This item was submitted to Loughborough's Research Repository by the author.

Items in Figshare are protected by copyright, with all rights reserved, unless otherwise indicated.

\title{
EcoPrinting: investigation of solar powered plastic recycling and additive manufacturing for enhanced waste management and sustainable manufacturing
}

\section{PLEASE CITE THE PUBLISHED VERSION}

https://doi.org/10.1109/sustech.2018.8671370

\section{PUBLISHER}

IEEE

\section{VERSION}

AM (Accepted Manuscript)

\section{PUBLISHER STATEMENT}

(c) 2018 IEEE. Personal use of this material is permitted. Permission from IEEE must be obtained for all other uses, in any current or future media, including reprinting/republishing this material for advertising or promotional purposes, creating new collective works, for resale or redistribution to servers or lists, or reuse of any copyrighted component of this work in other works.

\section{LICENCE}

All Rights Reserved

\section{REPOSITORY RECORD}

Mohammed, Mazher, Daniel Wilson, Eli Gomez-Kervin, Lucas Rosson, and Johannes Long. 2019.

"Ecoprinting: Investigation of Solar Powered Plastic Recycling and Additive Manufacturing for Enhanced Waste Management and Sustainable Manufacturing". Loughborough University.

https://hdl.handle.net/2134/12625496.v1. 


\title{
EcoPrinting: Investigation of Solar Powered Plastic Recycling and Additive Manufacturing for Enhanced Waste Management and Sustainable Manufacturing
}

\author{
Mazher Iqbal Mohammed*, Daniel Wilson, Eli Gomez-Kervin, Lucas Rosson and Johannes Long \\ School of Engineering, \\ Deakin University \\ Geelong, Australia \\ mazher.mohammed@deakin.edu.au
}

\begin{abstract}
In this article we propose the EcoPrinting technology, which aims at a near zero carbon foot print means of recycling waste polymers into functional, working products. To achieve this goal, we demonstrate a nanogrid device by which solar energy can be stored in a modest sized battery system and use this to power instrumentation for melt extrusion of waste polymers into 3D printer filaments. We then use this filament in a modified 3D printer system to manufacture functional humanitarian aid components such as water seals and pipe connectors. We investigate the feasibility of the EcoPrinting principal using ABS and HDPE plastics, while evaluating and optimizing enabling device energy consumption and manufacturing performance. We conclude that the EcoPrinting principal is possible and functional devices can be manufactured with mechanical integrity equivalent to commercially available components. We finally demonstrate that EcoPrinting can be used as a tool for humanitarian use, realizing a manufacturing paradigm that is self-sufficient and potentially capable of addressing challenges of plastic proliferation in developing nations.
\end{abstract}

Keywords-3D printing, Solar, Recycle, Humanitarian Aid, Plastic, Extrusion, Ecoprinting

\section{INTRODUCTION}

Plastics are a popular manufacturing material owing to their desirable material properties, such as their strength, durability and impact resistance. Equally, plastics are amenable to most commercial methods of mass manufacturing, such as injection molding [1], CNC machining [2] and 3D printing [3]. As such the use and production of plastics is escalating exponentially and is estimated to grow to a predicted 850 million tonnes each year in the lead up to 2050 [4]. This phenomenal volume of production is resulting in profound challenges regarding the management and recycling of this resource, particular in light of its non-biodegradability and diminishing material properties over time due to oxidative degradation [5]. The complexity of this issue is further exacerbated by the fact that plastics are predominantly synthesized using fossil fuels, and so production is expected to be perturbed in the near future. Therefore, it is becoming increasingly desirable to find flexible solutions whereby plastics can be reprocessed following the end of life. Equally, the ideal solution should not only be scalable, but accessible to the masses such that individuals as citizen scientists could play their part to help in this global challenge. 3D printing technology may be one such solution to address this challenge as it is a low cost and accessible technology, which in its most common form, uses plastics as its primary manufacturing material [5]. Additionally, recent studies have revealed that such platforms operate using relatively low average power (approximately $60 \mathrm{Whr}$ continuous use) feasibly allowing for a modest sized renewable energy system to power such technology [6]. If we combine both elements together, potential exists to create a low carbon foot print and sustainable means of energy generation to reprocess waste plastics into usable and functional items, a process we call EcoPrinting. Additionally, the low power consumption of 3D printer devices opens up the interesting possibility of using battery enabled renewable systems to make such technology portable [6]. In this study we examine the feasibly of creating a portable nanogrid style system, comprising a solar charging battery system, to power equipment that is capable of converting plastic waste found in the environment into end products. Several low power devices are devised to achieve this, comprising a hand operated granulation device, a low power filament extrusion system, a modified 3D printer amenable to use of the recycled plastics and a laptop for design development and 3D printer interfacing. As a working demonstration of the EcoPrinting system, we implement the technology as a means of providing humanitarian aid in the context of Water and Hygiene Sanitation (WASH) within remote regions of the Solomon Islands. In this environment we anticipate both a lack of stable electricity and equally an abundance of waste plastics, as confirmed by aid workers currently engaged in this region. In this study we will present results on how we make use of plastics found in the environment, which comprised ABS e-waste and discarded thermoformable hard plastics (High Density Polyethylene, HDPE), and convert these into 3D printer filaments. We then make use of this filament in our 3D printer system to create functional pipe connectors and plumbing seals to remedy failing water infrastructure in a jungle setting.

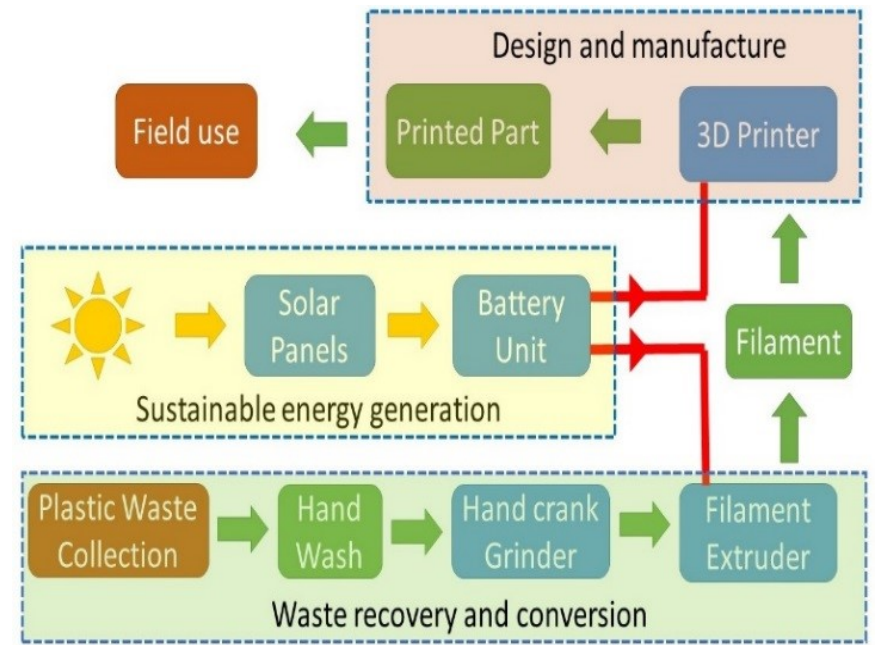

Fig. 1. Overview of the Ecoprinting concept for in field humanitarian use, highlighted the primary phases of waste recover and conversion, sustainable energy generation and manufacturing to create the final part suitable for field use. 


\section{DEVICE PRototype}

Our Ecoprinting system comprised four primary subcomponents, a hand operated grinding system, a custom built polymer filament extruder, a modified 3D printer and nanogrid power system. An overview of the different componentry and process stages can be seen in Figure 1.

\section{A. Waste Plastic Recovery and Processing}

In this study the Ecoprinting process had been optimised for the use of ABS plastics, but had potential to operate using HDPE. Plastics were recovered from various location within the city limits of Honiara, which is the capital city of the Solomon Islands. It was found that owing to a lack of any substantial recycling efforts on the island, waste plastics were found in abundance is most locations, and comprised plastics ranging across PET (plastic drinks bottles), HDPE/LDPE (jerry cans, detergent bottles, crates, etc) and ABS (electronic waste, car parts, etc). We focused our attention on examining three locations of interest, comprising the local landfill site, general waste found on the streets and waste found from local businesses. It was found that there was an abundance of candidate ABS plastics found in landfill and local businesses, generally in the form of e-waste.

\section{1) Waste Collection}
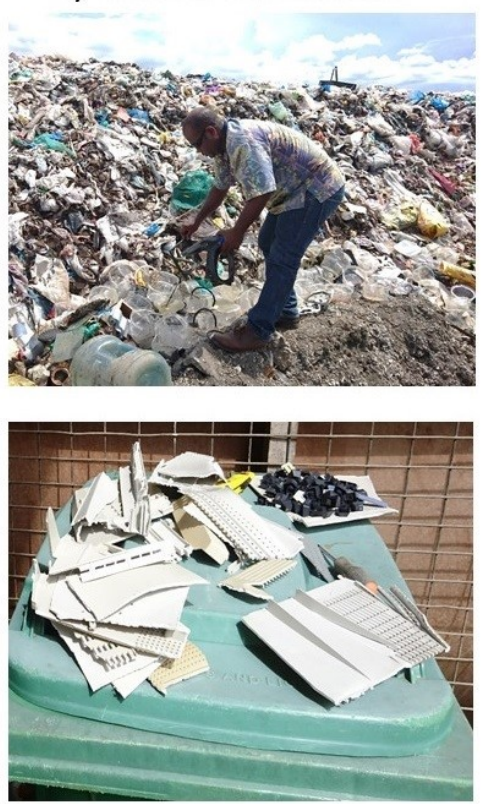

\section{3) Air dry and break down}

2) Dismantling
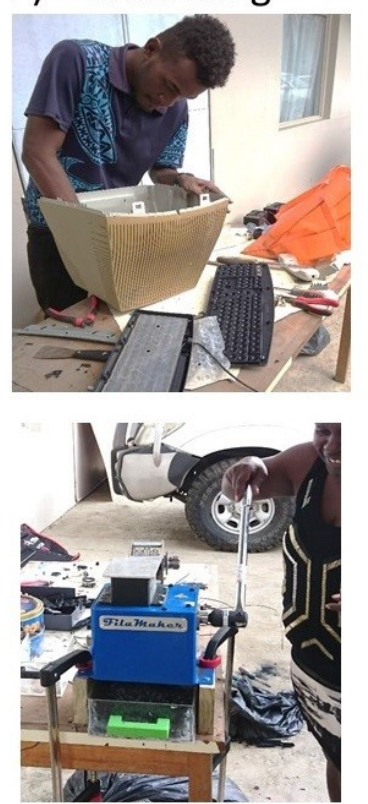

4) Hand grind
Fig. 2. An overview of the plastic waste recovery, preparation and granulation process.

To prepare the waste plastics for melt extrusion into filaments the initial phase is to dismantle the candidate waste, of all metallic and non-like plastic material. This operation was performed by hand, where we trained locals on the Solomon Islands on this procedure. Once plastics were separated, the parts were given a light wash to remove superficial dirt and grime, using bucketed water to minimize the waste of this precious resource. Once washed, the parts were allowed to air dry in the sun before they were ready for granulation. This methodology proved much easier to both dry the plastic and ensured that there were no issues with introducing moisture into the granulation device. Granulation was performed by breaking down the plastic parts into fragments that were approximately $150 \times 300 \mathrm{~mm}$ using a hacksaw, and were then manually fed into a hand operated grinder (Filamaker, Germany) to be broken down into granules. The grinder system was fabricated from hardened steel and could be operated using a geared long arm hand crank system, to allow for a person to hand perform the grinding operations. The grinding process was performed over multiple cycles, before the resulting fragments were separated using a custom made mesh device, with a mesh size of $4 \mathrm{~mm}$. Figure 2 shows the process to clean the plastic and create the source granulated plastic material.

\section{B. Energy Generation and Storage}

In the present system electricity was generated using a solar panel and lithium ion battery storage system, which we have named a nanogrid. The system was created to be dynamic such that the batteries could be simultaneously charged and discharged, allowing for the operation of a respective device and/or the charging of the batteries by the solar panels. The system comprised a commercial MPPT 75/15 charge controller (Victron, Outback Marine, Australia) used alongside two 12V 55Ahr LiFEPO4 batteries (EV Power, Australia) and two foldable $150 \mathrm{~W}$ silicon photovoltaic cell arrays (Australia Direct, Australia). The lithium-ion batteries were equipped with inbuild smart charge control systems ensuring the system did not over charge, or fully discharge, thereby guaranteeing the integrity of the system. An additional fuse and switch were placed into the system as a secondary safety precaution. To monitor the power entering the nanogrid system, an inline power meter was placed into the system (Australia Direct, Australia) which allowed for direct readings of the power, voltage and current.

Figure 3A) shows a picture of the final system built into a ruggedized casing for durability and ease of transport in the field. The particular set up was designed to operate at 24VDC due to both the 3D printer (bed/hotend heaters) and extruder (motor and heaters) components all operating at $24 \mathrm{~V}$. Therefore, to minimize losses due to voltage conversion, the system was designed to run seamlessly at $24 \mathrm{~V}$, however we retained secondary power supply units for devices to be run from the standard $240 \mathrm{~V}$ mains supply. This would allow for both devices to function in what was termed 'on grid', using mains electricity, or 'off grid', using the nanogrid system. To optimize the nanogrid set up, tests were also performed to measure the power consumption of each of the electrical devices used in the waste conversion and manufacturing process. To record the power consumption a Q4 Plus power meter (Metrel, Slovenia) was employed, which provides realtime current and voltage usage data.

\section{Waste Plastic Extrusion}

The granulated plastic products were converted into $3 \mathrm{D}$ printer filaments using a custom built thermal extruder device, we named the Ecostruder, which can be seen in figure $3 \mathrm{~b}$ ). The system is based on larger scale commercial units [7] and is powered by a $24 \mathrm{~V} 100 \mathrm{~W}$ geared motor and three $24 \mathrm{~V}, 50 \mathrm{~W}$ band heaters. The heaters were configured with one at the hopper to assist dehydration of the granulated material (set temperature $195^{\circ} \mathrm{C}$ ), one at the midpoint of the barrel (set temperature $200^{\circ} \mathrm{C}$ ) and the last heater at the extruder nozzle (set temperature $205^{\circ} \mathrm{C}$ ). A picture of the final system can be 
seen in figure 3B). To create filaments granulated plastics are fed into the hopper before being fed into a heated barrel, through three heating zones, before ejection from the extruder nozzle, which was fixed at $2.5 \mathrm{~mm}$. The feed of the material is controlled by a custom, tapered geometry drive screw, which was optimised for use with ABS plastic. As the granulated material migrates through the barrel, the polymer undergoes a phase change from solid to liquid. The change in screw geometry and the continuous introduction of new material into the barrel build up pressure in the barrel, leading to the evacuation of the liquid polymer through the nozzle in a continuous stream, forming the filament. The resulting filament is then cooled using a multi directional fan, before being fed into a sensor system to record the diameter. Based on a programmable diameter setting the system uses the filament sensor output to control a Proportional Integral Derivative (PID) controller, which provides feedback to the extruder drive screw speed to automate the diameter tolerance control. This feature is important as errors in the filament diameter results in inconsistencies during the 3D printing phase. The system also incorporates motor driven pulleys to help draw the resulting filament into an automated spooling system ready for use. Finally, the system has a user control interface such that any of the operational parameters can be adjusted, for maximum flexibility to optimise the end output of the filament diameter, or to process plastic materials other than ABS.

A)

Lithium Ion Batteries $\quad 3 D$ Printer Direct Wiring

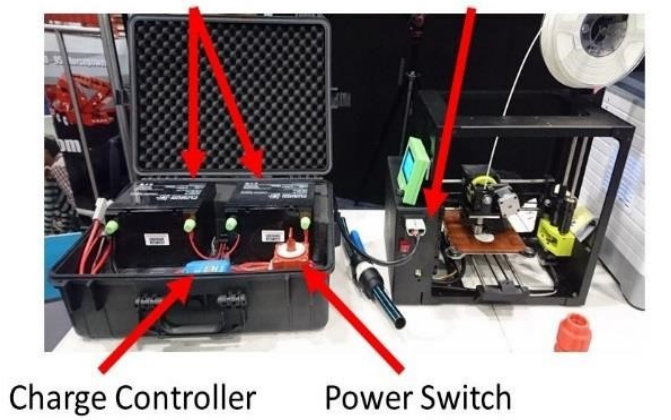

B)

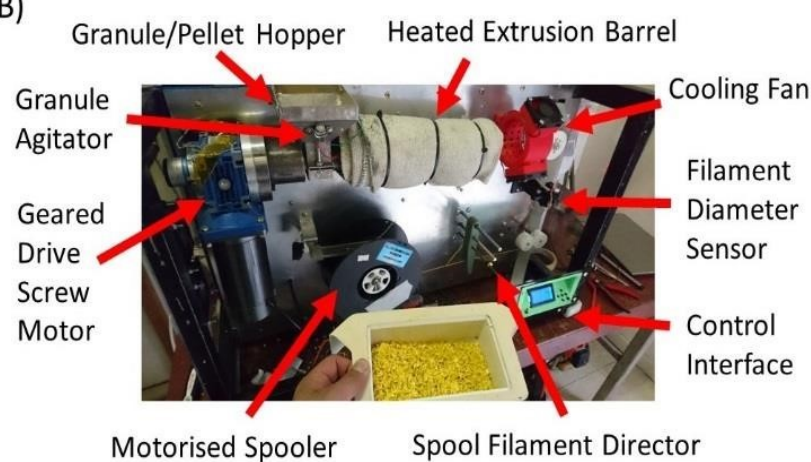

Fig. 3. A) Nanogrid battery unit with modified 3D printer and B) Filament Extruder system.

D. Design and $3 D$ printing with Waste The 3D printer system comprised a commercial open source printer system (Lulzbot Mini, Aleph Objects, USA) which underwent modifications to the printer hotend component to improve print melt flow characteristics when using recycled filaments. Additional modifications were made to the internal electrical components to allow the printer to operate from a $24 \mathrm{~V}$ power system to minimize energy losses due to voltage conversion. From initial testing the recycled ABS material was found to print with optimal printer parameters of nozzle temperature of $220^{\circ} \mathrm{C}$, print bed temperature of $95^{\circ} \mathrm{C}$ and print speed of $60 \mathrm{~mm} / \mathrm{s}$. When printing with HDPE material we found that the printer settings had to be altered to a nozzle temperature of $200^{\circ} \mathrm{C}$, print bed temperature left at room temperature and a print speed of $7 \mathrm{~mm} / \mathrm{s}$. Additionally, a layer of sacrificial HDPE material, in the form of a scavenged HDPE bottle, cut and spread flat, was required to improve the adhesion of HDPE, which would otherwise not adhere to the print bed. For all designs examined, prints were performed using $100 \%$ infill to ensure the fabrication of the most mechanically robust parts possible.

Humanitarian aid parts manufactured comprised a generic pipe connector design and a stem washer for water taps. All Computer Aided Designs (CAD) were created using Inventor 2018 (Autodesk, USA).

\section{RESULTS}

\section{A. Waste Plastic Granulation}

To manufacture the $3 \mathrm{D}$ printer filaments granulation is an important part of the process, in particular to control the size uniformity of the resulting fragments. The typical industry standard when manufacturing using either extrusion or molding technologies is to utilize pelletized form of a given polymer. This is because the pelletized product is more rounded in shape and equal in terms of average size, ultimately resulting in a more repeatable feed rate during extrusion. Therefore, it is important to achieve a size comparable to pelletized material to ensure predictable and even material flow during extrusion. To achieve this, the recycled plastics were processed over three repeated grinding cycles before being placed into the mesh to separate fragments based on size. This entire procedure was performed by hand thereby reducing energy requirements and we were able to realize granules with an average diameter of approximately $3.8 \pm 1.5 \mathrm{~mm}$, across a range of 2.6 and $5.2 \mathrm{~mm}$. This value compares favorably to the typical size of pellets, which has been reported to be approximately $3.4 \pm 0.4 \mathrm{~mm}$, across a range of 3.0 to $4.5 \mathrm{~mm}$ [6]. Assigning no energy cost in the processing of the granulated material, as everything is processes by person power, the main factor relating to the production of granulated plastics is the time taken to procure the plastics, alongside the processing time to dismantle, clean and granulate. We estimate that in this study using a three-person team, a time of approximately 45 hours was required to generate 4 kilograms of granulated plastics ready for processing into filament. By normal manufacturing standards this may be considered very time ineffective, however given the constraints in a developing nation setting where unemployment is high, using a localized workforce may be an effective means of sustaining such activities through job creation. Based on the national minimum wage being approximately SI $\$ 4.00$ per hour (approx. $\$ 0.50 \mathrm{USD})$, the cost to produce $4 \mathrm{~kg}$ of granulated material is estimated at \$7.50USD, based on 3 people working for 5 hours. It should be noted that if the work force are volunteers or otherwise unemployed personnel, which is often the case in remote areas of the Solomon Islands, this cost is effectively zero. 


\section{B. Energy Usage and Generation}

To guide the design of the nanogrid system, tests were performed to understand the power consumption from both the Ecostruder and 3D printer systems. Measurements were performed of the two system during usage from a test battery unit, to simulate power consumption from the nanogrid system. Figure $4 \mathrm{~A}$ and $4 \mathrm{C}$ ) illustrate the real-time power consumption by the Ecostruder and $3 \mathrm{D}$ printer. It was found that the Ecostruder, over a warm up period of approximately 28 minutes and continuous operation of 38 minutes had an average continuous power usage of $137 \mathrm{~W}$ and an average energy consumption of $151 \mathrm{Wh}$. The $3 \mathrm{D}$ printer system was measured to have an average continuous power usage of $121 \mathrm{~W}$ and average energy consumption of $34.3 \mathrm{Wh}$ over a 18 minute run time printing a test object. Based on these findings we estimated that over a typical working day with approximately 2 hours run time of the ecostruder and 8 hours run time for two $3 \mathrm{D}$ printers, and taking into account an $85 \%$ compensation factor to account for power usage of the charge controller and other system based losses, we would use a total of approximately $1000 \mathrm{Wh}$. Given the system runs using a $24 \mathrm{~V}$ supply, we estimate $41.7 \mathrm{Ah}$ usage over a typical day. We therefore opted for a system with a total charge capacity of $55 \mathrm{Ah}$ and $300 \mathrm{~W}$ solar panel system for charge generation, so that we had sufficient charge to accommodate daily fluctuation in energy consumption, in addition to extended periods where charge is not generated.

A)
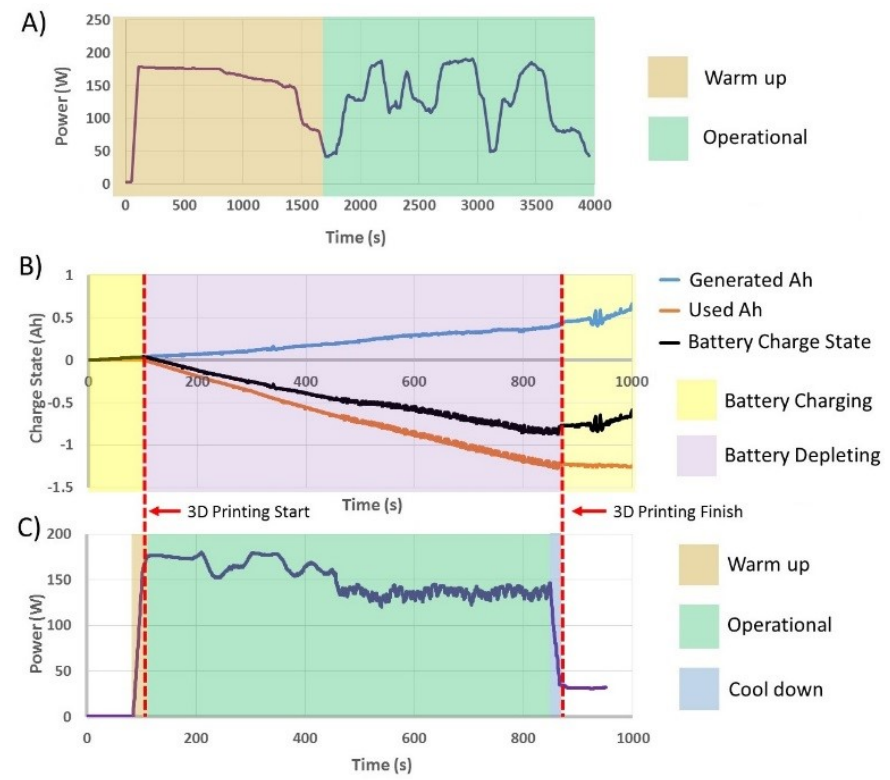

Fig. 4. A) Real-time power usage during the warm up and usage of the Ecostruder filament generation device, B) A graph showing a comparison of the Ah discharging and charging from the nano grid system during real-time $3 \mathrm{D}$ printing and charge generation by the solar panels. C) Real-time power consumption of the 3D printer system during the recording of the data in $\mathrm{B}$ ).

Tests were also performed to examine the charge generation and depletion dynamics of the nanogrid system. The results of one test can be seen in figure 4B), where the positive data curve illustrates the charge generated by the solar panels against the lower negative curve illustrated the charge depletion due to use of the 3D printer system. The resulting middle curve illustrates the charge state of the battery relative to its starting charge. With respect to the battery, it can be seen overall the charge is depleted by approximately $0.7 \mathrm{Ah}$ after continuous printing for a 13 minute period. However, following printing completion when the system become idle, the solar panels begin to regain the lost charge, resulting in a net loss of approximately $0.55 \mathrm{Ah}$ over a 16.6 -minute test period.

Owing to the limitations on in field operation, we did not have the capacity to perform real-time continuous data logging when on the Solomon Islands. However, we implemented an in line power meter to monitor the output power from the solar panels of the nanogrid system. During operation we recording a peak power generation of approximately 200W, which indicated an excess of power generation. Equally, we recorded instances where the power generation had dropped to $0.1 \mathrm{~W}$, which was far less than that required to operate the system in real time. Therefore, our assumption to implement a battery system far in excess of anticipated daily Ah usage adequately supplemented the system during times where energy generation is low. During the project we did not experience a time when we did not have sufficient power to run the $3 \mathrm{D}$ printer, thereby avoiding downtime issues with the manufacturing of aid components.

\section{Filament Extrusion}

The plastic granules were then loaded into Ecostruder hopper to begin production of the filaments. We fixed the system to produced filament with a $2.2 \mathrm{~mm}$ diameter, which from in-house testing proved to be the most stable diameter achievable by the system (results not shown). The granulated plastics are coarser in size and shape than those of commercial pelletized material. This can result in uneven flow from the hopper into the extruder barrel, resulting in more erratic fluctuations in the feed rates during extrusion. In worst cases the flow of material can completely halt due to blockages of the material in the hopper. To combat this issue, we introduced a mechanical agitator device, which continuously vibrated the extruder hopper. Using this device, no blockages were observed using the granulated plastics.

The system was primarily optimized to use ABS plastics which were sourced from e-waste, comprising computers, keyboards and inkjet printer cartridges. When examining the granulated material, it was found that some samples appeared to be in a near new condition, however, some recovered plastics looked discolored. We believe this discoloration was a sign of UV/oxidative damage ABS polymer. When extruded the discolored material was found to have a low viscosity and did not hold its form, as ABS would typically would. Additionally, when the resulting material did hold its form, it was found to have many imperfections along the length of the filament. We therefore disregarded much of this material and focused extrusion on material that was not discolored. When extruding this ABS material, we found the resulting filaments were of a similar consistency to filaments generated using pelletized material, with a smooth surface finish, free of defects, and with an ability to hold its form post extrusion.

The extruder system was designed to incorporate several embedded sensors to monitor performance, this included temperature on each heater, the filament diameter and the rotation speed of the drive motor. Figure 5 illustrates recorded outputs from the temperature probe on central heater, set at $200^{\circ} \mathrm{C}$, and the filament diameter over a period of $400 \mathrm{~s}$. Continuous monitoring of the temperature outputs revealed a stability within $2-3^{\circ} \mathrm{C}$, which was improved during the trip to 
$1-1.5^{\circ} \mathrm{C}$ through optimization of the PID controller. Examination of the generated filament revealed an average diameter of $2.19 \pm 0.08 \mathrm{~mm}$ which is close to the tolerances found in commercial filaments, which are typically within $\pm 0.05 \mathrm{~mm}$.
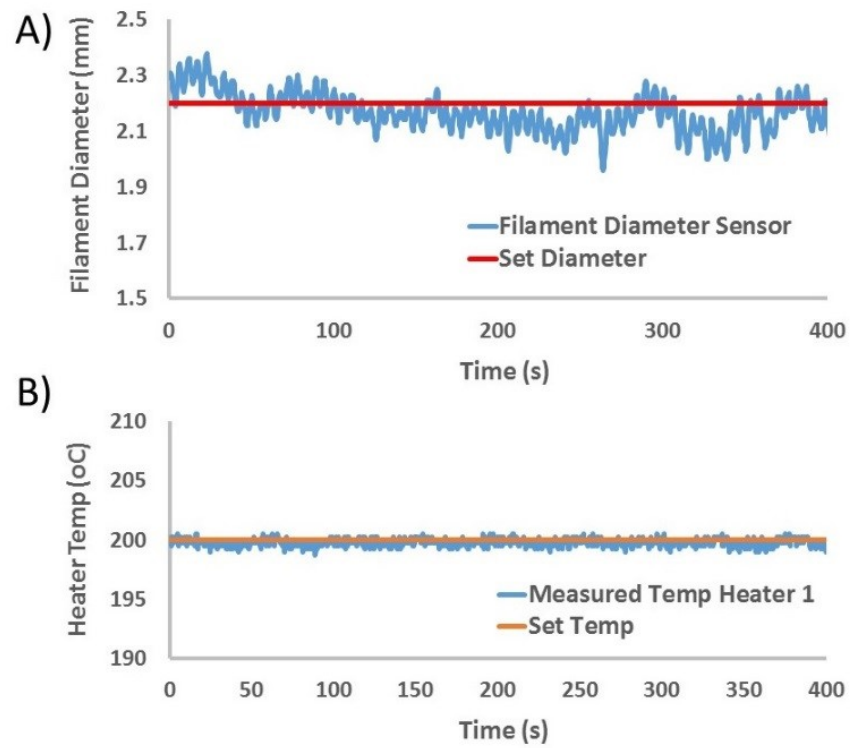

Fig. 5. A) A graph of the recorded output from the filament diameter sensor with a line indicated the set diameter of $2.2 \mathrm{~mm}$ and B) A graph illustrating the fluctuation of the temperature probe on heater two of the extruder with a line illustrating the set temperature of $200^{\circ} \mathrm{C}$.

The extruder feed screw was set to rotate at a speed of approximately $25-30 \mathrm{rpm}$, allowing for the production of $1 \mathrm{~kg}$ of filament in approximately 1-1.5 hrs. Unfortunately, during the extrusion process there are losses of material which inevitably occur due to the priming of the system and poorly formed filaments while the closed loop control system stabilizes. This wastage was approximated to be $3040 \mathrm{~g}$ for every $\mathrm{kg}$ that is extruded. Therefore, the production capacity of the system is $0.96 \mathrm{~kg}$ every $1.5 \mathrm{hrs}$, with approximately $4 \%$ loss of source material. This can also be equated to a loss of $6.04 \mathrm{Wh}$ of energy by the extruder due to manufacturing inferior end products. For this project approximately $4 \mathrm{~kg}$ of ABS filament was generated, and so we approximate a maximum loss of $160 \mathrm{~g}$ of ABS material and $24.16 \mathrm{Wh}$ of energy. Ultimately, such losses are inevitable to the extrusion process and so considered acceptable losses.

Beyond the extrusion of ABS, we also demonstrated the conversion of a discarded HDPE jerry can. This plastic was extruded in a similar fashion to the ABS however on this occasion the temperature for each heater was reduced by $20^{\circ} \mathrm{C}$, while all other extruder settings were kept fixed. This filament was found to be more inconsistent in terms of end dimeter, fluctuating between approximately 1.6 to $3.5 \mathrm{~mm}$. We hope to refine the extrusion of was HDPE further in future studies.

Following extrusion, an initial test was performed to ensure the integrity of the produced filaments. Print tests revealed similar performance to that of commercially available filaments, and optimal printing was achieved using a print speed of $60 \mathrm{~mm} / \mathrm{s}$, hotend temperature of $220^{\circ} \mathrm{C}$ and print bed temperature of $95^{\circ} \mathrm{C}$.

\section{Humanitarian Aid Design and 3D Printing}

Following the production of the $3 \mathrm{D}$ printer filaments a scoping study was performed in the village of Visale, which some $30-40 \mathrm{~km}$ from Honiara, in a remote area of jungle. The village was selected as it was disconnected from any mains electricity infrastructure, whilst also being in desperate need for humanitarian solutions for failing water infrastructure. Upon initial investigation in the village it was found there was approximately 12-14 substantial leaks ranging from the necessity to repair whole sections of piping, sections requiring a pipe connector or taps which were leaking due to a lack/perished seals. Given that 3D printing process is better suited to smaller parts with high levels of design complexity, we opted to leverage the system to create various pipe connectors and seals for the leaking taps.

For the pipe connector sections, a basic measurement of the outer diameter of the leaking pipes was performed. In all instances examined, the pipes were generally of two different sizes, due to the residents making modifications with whatever resources were at hand. The consequence of this was that necessary sizes were generally not available as

'off-the-shelf' components, and so the use of 3D printing with its mass customization potential was ideal to address such a challenge.

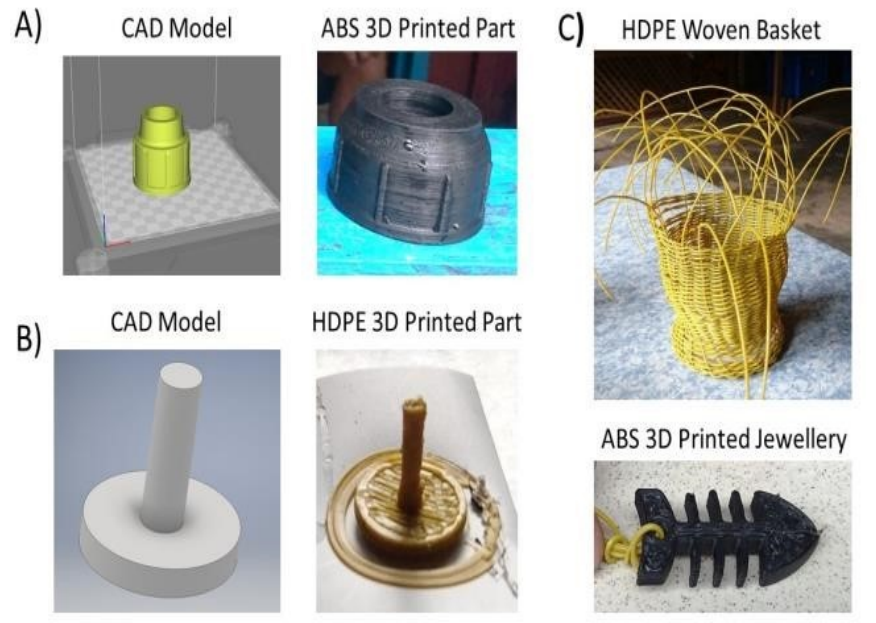

Fig. 6. $\mathrm{CAD}$ and $3 \mathrm{D}$ printed parts for a) and $\mathrm{ABS}$ pipe connector cap and B) a HDPE step circular tap seal. C) Examples of additional product that were possible using recycled plastic materials, comprising a basket woven from HDPE filaments and an ABS printed piece of jewellery, with a HDPE filament pendant string.

Several CAD concepts were prepared to address individual challenges found relating to remediation of the failing water infrastructure. Figure 6 illustrated some of the devised components, including a pipe connector cap and a stem seal for a tap. Scrutinizing the parts more closely it can be seen that the cosmetic finish on the outer surfaces of the parts are arguably inferior to commercially available parts. However, in this particular instance, the cosmetic appearance was not important and functionality was the most important facet. It is also noted that another advantage of $3 \mathrm{D}$ printing is the ability to adjust a parts geometrical attributes to match a particular situation or desired performance attribute, such as mechanical strength. In this instance, water infrastructure parts were made to be larger than their commercial equivalents to provide the greatest possible mechanical integrity to avoid failure. Despite 
this requiring more material and energy to fabricate, it was considered an acceptable compromise given the enhanced health implications of remedying issues relating to access of drinking and sanitation based water supply.

Figure 7 illustrates one of the leaking pipes within the jungle village that was selected for remedying. A pipe connector was fabricated to the exacting pipe outer diameter and manufactured from the recycled plastic filament. Following two iterations successful components were manufactured, which completely eliminated all leaks, as can be seen in figure 7 .
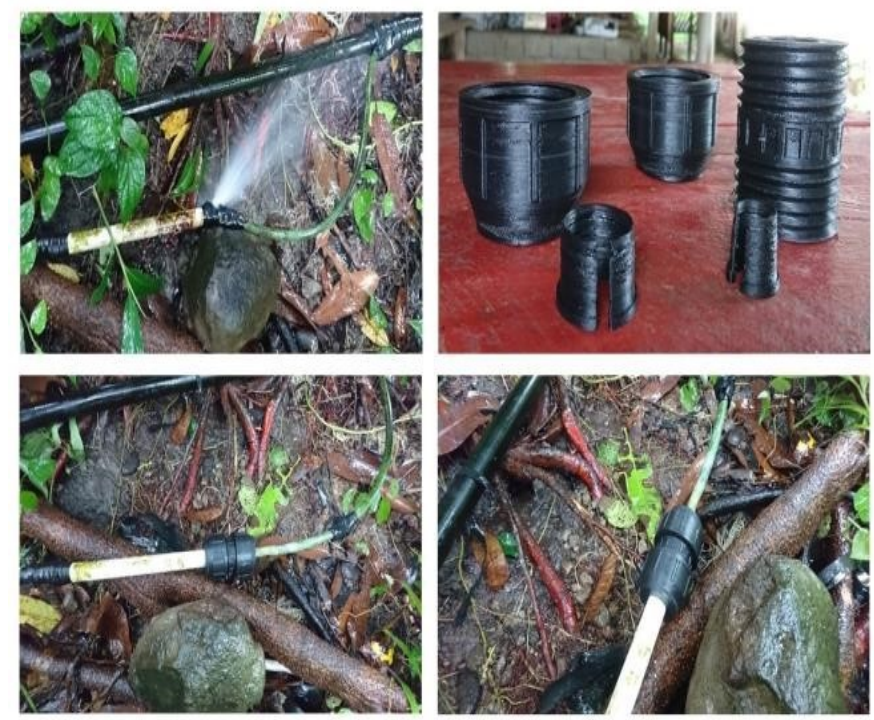

Fig. 7. Leaking and fixed water pipe and the $3 \mathrm{D}$ printed pipe connector made from recycled plastic

The pipe connector seen in figure 7 was estimated to have taken approximately 9 hours to fabricate and comprised approximately $700 \mathrm{~g}$ of $\mathrm{ABS}$ material. We estimate the energy cost for fabrication to be approximately $234 \mathrm{Wh}$, which could be recovered by the nanogrid system within less than $2.5 \mathrm{hrs}$. The tap seal, which can be seen in figure 6B, took approximately 20 minutes to fabricate, requiring approximately $8 \mathrm{Wh}$ to produce. Interestingly, beyond the production of humanitarian supplies, we also demonstrated wider manufacturing opportunities, fabricating a woven basket and jewellery. Both items are popular products found on the island, often made by the indigenous population. This hints at the wider potentials of the system to enable culturally significant and motivated products manufacturing and we hope to explore this opportunity in future work.

\section{DISCUSSION}

The devised Ecoprinting system was deployed in the Solomon Islands, on the island of Guadalcanal to test its efficacy in real conditions. It was found that the devised system worked efficiently using the nanogrid system allowing for continuous usage with excess energy generation over the trial period for both filament extrusion and 3D printing. The recycled plastics could be formed into filaments with tolerances equal to those of commercial grade filaments. Using this filament, we could manufacture functional humanitarian aid supplies to solve leakages in water pipes alongside functional seals for taps. On the Solomon Islands a single pipe connector can cost as much as \$150USD. We therefore estimate our system beyond its initial outlay and operator time could pay for itself after forming approximately 53 pipe connectors, which could be achieved in less than 2-3 months. We believe the system could readily be scaled for volume production with multiple systems, or function as a standalone, portable tool with near unlimited design potential and which not only promotes sustainable practices but could realistically transform humanitarian aid delivery as we know it.

\section{Acknowledgements}

We would like to thank both the Deakin University School of Engineering and Centre for Humanitarian Leadership, alongside Plan International for their support and funding for the project. We would like to thank the English Foundation and members of the general public, who provided much needed financial support to the project following crowd funding efforts on the Start Some Good 3DWASH campaign. We would finally like to thank James Lamont and Michael Shanahan for their valuable technical support in the project.

\section{REFERENCES}

R. A. Malloy, Plastic part design for injection molding: Hanser Publishers New York, 1994.

A. Boschetto, L. Bottini, and F. Veniali, "Finishing of Fused Deposition Modeling parts by

CNC machining," Robotics and Computer Integrated Manufacturing, vol. 41, pp. 92-101, 2016/10/01/ 2016.

[3] M. I. Mohammed and I. Gibson, "Design of Three-Dimensional, Triply Periodic Unit Cell Scaffold Structures for Additive Manufacturing," Journal of Mechanical Design, vol. 140, p. 071701, 2018.

[4] M. A. Kreiger, M. L. Mulder, A. G. Glover, and J. M. Pearce, "Life cycle analysis of distributed recycling of post-consumer high density polyethylene for 3-D printing filament," Journal of Cleaner Production, vol. 70, pp. 9096, 2014/05/01/ 2014.

[5] M. I. Mohammed, A. Das, E. Gomez-Kervin, D. Wilson, and I. Gibson, "EcoPrinting: Investigating the use of $100 \%$ recycled Acrylonitrile Butadiene Styrene (ABS) for Additive Manufacturing," in Proceedings of the 28th Annual International Solid Freeform Fabrication Symposium, 2017, pp. 532-542.

[6] M. I. Mohammed, M. Mohan, A. Das, M. D. Johnson, P. S. Badwal, D. McLean, et al., "A low carbon footprint approach to the reconstitution of plastics into 3D-printer filament for enhanced waste reduction," $\mathrm{KnE}$ Engineering, vol. 2, pp. 234-241, 2017.

[7] G. J. Listner and A. J. Sampson, "Single screw extruder," ed: Google Patents, 1970. 